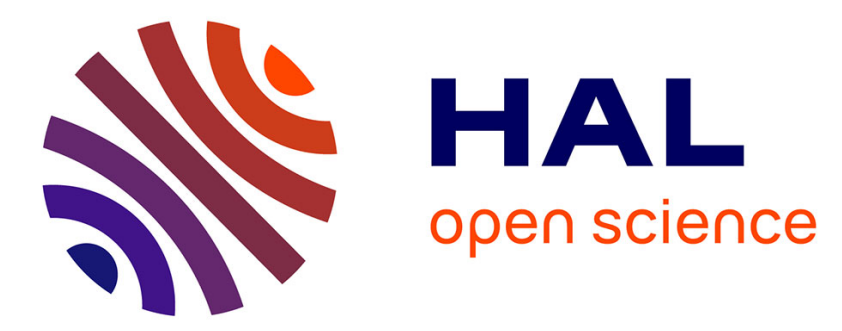

\title{
Concurrent data transmissions in green wireless networks: When best send one's packets?
}

Matthieu de Mari, Romain Couillet, Emilio Calvanese Strinati, Mérouane Debbah

\section{- To cite this version:}

Matthieu de Mari, Romain Couillet, Emilio Calvanese Strinati, Mérouane Debbah. Concurrent data transmissions in green wireless networks: When best send one's packets?. IEEE ISWCS 2012, Aug 2012, Paris, France. pp.596 - 600, 10.1109/ISWCS.2012.6328437 . hal-00769469

\section{HAL Id: hal-00769469}

https://hal-centralesupelec.archives-ouvertes.fr/hal-00769469

Submitted on 8 Jan 2013

HAL is a multi-disciplinary open access archive for the deposit and dissemination of scientific research documents, whether they are published or not. The documents may come from teaching and research institutions in France or abroad, or from public or private research centers.
L'archive ouverte pluridisciplinaire HAL, est destinée au dépôt et à la diffusion de documents scientifiques de niveau recherche, publiés ou non, émanant des établissements d'enseignement et de recherche français ou étrangers, des laboratoires publics ou privés. 


\title{
Concurrent data transmissions in green wireless networks: when best send one's packets ?
}

\author{
Matthieu De Mari*, Romain Couillet*, Emilio Calvanese Strinati ${ }^{\dagger}$ and Mérouane Debbah* \\ * Dept. of Telecommunications - Alcatel-Lucent Chair, SUPELEC, France, Email: firstname.lastname@supelec.fr \\ $\dagger$ CEA Leti, France, Email: emilio.calvanese-strinati@cea.fr
}

\begin{abstract}
In this paper, we consider the scenario of a cellular network where base stations aim to transmit several data packets to a set of users in the downlink, within a predefined time, at minimal energy cost. The base stations are non-cooperating and the instantaneous transmission rate depends on the instantaneous SINR at the receiver. The purpose of this article is to highlight a power-efficient transmit policy. By assuming a large number of homogeneous users, we model the problem as a mean field game, with tractable equations, that allow us to bypass the complexity of analyzing a Nash equilibrium in a L-body dynamic game. The framework we propose yields a consistent analysis of the optimal transmit power strategy, that allows every base station to, selfishly but rationally, satisfy its transmission, at a minimal energy cost.
\end{abstract}

\section{INTRODUCTION}

Power control problems have become a relevant issue and a challenge for multi-user communications and green powerefficient networking [1][2]. In this article, we propose a scenario of transmission in a multi-cell network and aim to study a power-efficient transmission policy for Base Stations (BSs). More precisely, we seek transmission strategies that will improve the energy-efficiency of the network, i.e. that will reduce the transmit power consumption of every BS, while guaranteeing transmissions to be achieved in an acceptable time, for every user. Similar scenarios and studies of power adjustment and optimization, under a data rate constraint, have recently been explored. In [3], under a constraint of QoS, the power level of each user is adjusted in order to minimize the power consumption of every user in the uplink. In [4], still in the uplink, every user wants to distribute its power in order to maximize its own data rate, over a finite time-horizon.

In this paper, we propose a scenario of downlink transmission, consisting of a set of BSs, which are required to transmit a certain amount of data to a set of user terminals (UTs), in an acceptable time period. Every BS is acting selfishly: the BSs wish to minimize their transmissions cost individually. The BS compete and interfere: every BS has to achieve a transmission within a certain time and the transmissions of every BS will interfere at the non-intended UTs. The instantaneous datarate at each receiver then depends on by the instantaneous transmit power of every BS, as well as the interference from other BSs. The objective for each BS is to selfishly minimize its total energy usage while achieving data reception, by taking into account the other BSs' policies. This constrained concurrent power minimization problem under a constraint of data packet transmission can be defined as a dynamic game. Non-cooperative dynamic game theory provides tools to solve this problem [6]. We seek here a Nash equilibrium of the game, i.e. a transmit policy for which none of the users wants to change its strategy, since any deviation from the optimal power consumption might lead to a higher power consumption. Moreover, we will assume the channels between the BSs and the UTs to be time-varying and modelled according to an Itô process [10]. The stochastic dynamic game model however leads to intractable solutions, when it comes down to solving multi-user problems : in a multi-user case, the study of a Nash equilibria becomes complex.

However, recent works on Mean Field Game (MFG) theory have provided interesting results to deal with the asymptotic case, where the number of users grows to infinity [8][9]. When the number of users grows large, supposing a certain symmetry of the system model, we can replace the individual interactions to any user by an average overall interaction, called the mean field. This way, we can turn a multi-user problem into a singleuser problem, easier to solve (since every user is by symmetry representative of the whole population). The MFG theory leads in general to solving a couple of differential equations, known as Hamilton-Jacobi-Bellman (HJB) and Fokker-PlanckKolmogorov (FPK) equations, that greatly simplify the study of the Nash equilibria. It also brings sufficient insight about the approximate behavior of every user in more realistic systems. In [5], we find an application example of the MFG theory, to optimize the power consumption of electrical and hybrid vehicles in smart grids. As this is in general very technical to prove, we will take as granted that solutions to the couple of equations (HJB and FPK) exist and that they are consistent with the system. Mathematical discussions on this topic can be found in [8][9][11]Simulations will confirm that such solutions to our problem may exist.

The paper is structured as follows. In sec. II-A, we introduce the system model. In sec. II-B, we introduce the optimization problem. In sec. III, we provide details on the analysis of a Nash equilibrium in a L-user stochastic differential game. The sec. IV provides the fundamental equations to be used for analyzing a Nash equilibrium in the MFG. In sec. V, we provide simulation results for a simple scenario, where all the channels are no longer time-varying, but assumed constant and equal. 


\section{SYSTEM AND PROBLEM DEFINITION}

We consider a downlink narrow-band system consisting of $L$ BSs and $L$ UTs. We assume that UT $l$ is associated to one and only one BS $l$. The BS $l$ is required to transmit a data packet of size $Q_{l}$ to UT $l$ within a time period $T$. We define $Q_{l}(t)$ the size of the data packet which remains at time $t$, for UT $l$. In particular, the initial packet size is $Q_{l}(0) \triangleq Q_{l}$. We denote $h_{i l}(t)$ the channel between BS $i$ and UT $l$ at time t. $z_{l}(t)$ refers to the noise at UT $l$ at time $t$, assumed i.i.d. centered Gaussian with variance $\sigma_{n}^{2}$. The received signal $y_{l}(t)$ at $\mathrm{UT} l$ at time $t$ is given by:

$$
y_{l}(t)=\sum_{i=1}^{L} h_{i l}(t) x_{i}(t)+z_{l}(t)
$$

where $x_{l}(t)$ refers to the transmitted symbol by BS $l$. We define the transmit power from BS $l$ at time $t$ by $p_{l}(t)=$ $\mathbb{E}\left[x_{l}(t) x_{l}^{*}(t)\right]$. We also denote $p(t)=\left(p_{1}(t), \ldots, p_{L}(t)\right)$.

The channel dynamics are modeled stochastically [12] :

$$
d h_{i l}(t)=\underbrace{\left(a_{i l}(t) h_{i l}(t)+b_{i l}(t)\right)}_{\alpha_{i l}\left(t, h_{i l}(t)\right)} d t+\sigma_{b} d W_{i l}(t)
$$

This evolution law consists of a deterministic part $\alpha_{i l}\left(t, h_{i l}(t)\right)$ which accounts for both path loss or shadowing, and a random part which accounts for both fast fading and channel uncertainty $\left(d W_{i l}(t)\right.$ stands for mutually independent Wiener processes and $\sigma_{b}$ is positive)

An important metric here is the instantaneous transmission data rate $w_{l}(t, p(t), h(t))$ by $\mathrm{BS} l$, defined by:

$$
\begin{aligned}
& w_{l}(t, p(t), h(t))=B \ln \left(1+\gamma_{l}(t, p(t), h(t))\right) \\
& \gamma_{l}(t, p(t), h(t))=\frac{p_{l}(t)\left|h_{l, l}(t)\right|^{2}}{\sigma_{n}^{2}+\sum_{i \neq l}^{L}\left|h_{i, l}(t)\right|^{2} p_{i}(t)}
\end{aligned}
$$

where $B$ is the signal bandwidth and $h(t)=$ $\left(h_{11}(t), h_{12}(t), \ldots, h_{i l}(t), \ldots, h_{L L}(t)\right) . \quad \gamma_{l} \quad$ refers to the Signal to Interference plus Noise Ratio (SINR) at UT $l$. The evolution of the remaining packet size is then given by:

$$
d Q_{l}(t)=-w_{l}(t, p(t), h(t)) d t .
$$

Under some mild conditions on $a_{i l}, b_{i l}$ and $\sigma_{b}$, the initial conditions on the packets sizes $Q_{l}(0)$, the initial channels $h_{i l}(0)$ and the evolution laws (2) and (3) will lead to a unique trajectory for $Q_{l}(t)$ and $h_{i l}(t)$ [7]. We assume these conditions to be met.

The objective of this article is to determine the transmit power strategy $p_{l}(t)$ for $\mathrm{BS} l$, in a bounded action set $\left[0, P_{\max }\right]$ which allows BS $l$ to transmit its full packet $Q_{l}(0)$ to UT $l$, within duration $T$ (a complete transmission for BS $l$ will then induce $Q_{l}(T)=0$ ) and that will minimize the total consumed energy. The scenario we consider can then be modelled according to the following minimization problem:

$$
\min _{\left(p_{l}(t)\right)} \mathbb{E}\left[\int_{0}^{T} p_{l}(u) d u+K\left(Q_{l}(T)\right]\right.
$$

where $K\left(Q_{l}(T)\right)$ is the final penalty cost function for not achieving a complete transmission for user $l$ within time $T$, and $\int_{0}^{T} p_{l}(t) d t$ is the total consumed energy by BS $l$ on the time window $[0, T]$. The problem is constrained by the system dynamics:

$$
\begin{aligned}
d X(t) & =f(t, p(t), h(t)) \cdot d t+F \cdot d W_{t} \\
X(t) & =(Q(t), h(t))^{T} \text { and } Q(t)=\left(Q_{1}(t), \ldots, Q_{L}(t)\right)
\end{aligned}
$$

with

$$
\begin{array}{r}
f(t, p(t), h(t))=\left(-w_{1}(t, p(t), h(t)), \ldots,-w_{L}(t, p(t), h(t)),\right. \\
\left.\alpha_{11}\left(t, h_{11}(t)\right), \ldots, \alpha_{L L}\left(t, h_{L L}(t)\right)\right)^{T}
\end{array}
$$

and $F$ is the $L(L+1) \times L(L+1)$ diagonal matrix:

$$
F=\operatorname{diag}(\underbrace{0, \ldots, 0}_{L \text { times }} \sigma_{11}(t), \ldots, \sigma_{L L}(t))
$$

for $Q_{l}(0)$ and $h_{i l}(0)$ fixed.

\section{THE L-USER DYNAMIC STOCHASTIC GAME}

In order to solve the minimization problem above, let us first define the running cost function for UT $l, v_{l}\left(t, X_{t}\right)$ :

$$
v_{l}\left(t, X_{t}\right)=\mathbb{E}\left[\int_{t}^{T} p_{l}(u) d u+K\left(Q_{l}(T)\right)\right]
$$

A power strategy $p^{*}(t)=\left(p_{1}^{*}(t), \ldots, p_{l}^{*}(t)\right)$ is a Nash equilibrium for the dynamic stochastic game if and only if $\forall l, p_{l}^{*}(t)$ is an optimal admissible control to the problem:

$$
\min _{p_{l}(t)} \mathbb{E}\left[\int_{t}^{T} p_{l}(u) d u+K\left(Q_{l}(T)\right)\right]
$$

such that $\forall k \neq l, p_{k}(t)=p_{k}^{*}(t)$.

In our case, a Nash equilibrium will consist of a set of power strategies $p^{*}(t)$, where BS $l$ does not want to deviate from its power strategy $p_{l}^{*}(t)$, since it would result in a worse configuration for UT $l$. From now on, we denote the optimal trajectories of the running cost functions related to the Nash equilibrium $p^{*}(t)$, by $v^{*}\left(t, X_{t}\right)=\left(v_{1}^{*}\left(t, X_{t}\right), \ldots, v_{L}^{*}\left(t, X_{t}\right)\right)$. According to [6][7], a sufficient condition for the existence of a Nash equilibrium is the existence of a joint solution $v\left(t, X_{t}\right)$ to the $L$ Hamilton-Jacobi-Bellman(HJB) equations:

$$
\begin{gathered}
\min _{p_{l}(t)}\left[p_{l}(t)+f(t) \partial_{X} v_{l}\left(t, X_{t}\right)+\frac{1}{2} \operatorname{tr}\left(F^{2} \partial_{X X} v_{l}\left(t, X_{t}\right)\right)\right] \\
+\partial_{t} v_{l}\left(t, X_{t}\right)=0
\end{gathered}
$$

where we denote $\partial_{X} v\left(t, X_{t}\right)$ (respectively $\partial_{X X} v\left(t, X_{t}\right)$ ) the first (resp. second) order partial derivative of the function $v\left(t, X_{t}\right)$ with respect to $X$. The differentiation $\partial_{X} v\left(t, X_{t}\right)$ is in our case the gradient of $v\left(t, X_{t}\right)$ with respect to all the packet sizes $Q_{l}(t)$ and all the channels $h_{i l}(t)$. And the differentiation $\partial_{X X} v\left(t, X_{t}\right)$ is the Laplacian matrix. More precisely, the HJB equation for UT $l$ reads:

$\partial_{t} v_{l}\left(t, X_{t}\right)+\min _{p_{l}(t)}\left[p_{l}(t)-\sum_{k} w_{k}(t, p(t), h(t)) \partial_{Q_{k}} v_{l}\left(t, X_{t}\right)\right.$ 


$$
\left.+\sum_{i, k} \alpha_{i k}(t) \partial_{h_{i k}} v_{l}\left(t, X_{t}\right)+\sum_{i, k} \frac{1}{2} \sigma_{b}^{2} \partial_{h_{i k} h_{i k}} v_{l}\left(t, X_{t}\right)\right]=0
$$

A condition for the existence of a solution $v^{*}\left(t, X_{t}\right)$ to the previous system of HJB equations (5), is discussed in [11]. We will suppose that, in our problem, the functions are wellchosen, so this condition is met. The optimal transmission policies $p^{*}\left(t, X_{t}\right)$ can be obtained from $v^{*}\left(t, X_{t}\right)$ :

$$
\begin{aligned}
& \forall l, p_{l}^{*}\left(t, X_{t}\right)=\arg \min _{p_{l}(t)}\left[p_{l}(t)+\sum_{i, k} \alpha_{i k}(t) \partial_{h_{i k}} v_{l}^{*}\left(t, X_{t}\right)\right. \\
& \left.-\sum_{k} w_{k}(t, p(t), h(t)) \partial_{Q_{k}} v_{l}^{*}\left(t, X_{t}\right)+\sum_{i, k} \frac{1}{2} \sigma_{b}^{2} \partial_{h_{i k} h_{i k}} v_{l}^{*}\left(t, X_{t}\right)\right]
\end{aligned}
$$

We have assumed the existence of optimal transmission policies to the L-body stochastic dynamic game and put in evidence the equations to be solved, in order to analyze a Nash equilibrium of the game. The resolution of the coupled HJB equations is however complex when $L$ is greater than two. We will simplify our problem by assuming that the number of agents $L$ becomes extremely large and that each user is alike. For this, we will turn the $L$-body game formulation into a mean field game.

\section{A MEAN FIELD APPROACH}

Recent works on MFG [8][9] have shown interesting results when the number of users in the game is large enough and these users are sufficiently indistinguishable. In ths setting, from one BS's point of view, it becomes equivalent to consider all the other transmissions as a continuum. Therefore only the distribution of the other states is needed for one BS to take into account the other BSs' actions. This greatly simplifies the problem: we will obtain only two coupled differential equations (a HJB equation and a FPK), instead of $L$ HJB equations, that will determine the mean field equilibrium of the game. In the previous game, the impact of all the BS except BS $l$ on one UT $l$ decisions was expressed through the interference term $I_{l}\left(t, X_{t}\right)$ :

$$
I t_{l}\left(t, X_{t}\right)=\sum_{k \neq j}\left|h_{k l}(t)\right|^{2} p_{k}(t)
$$

If we assume similar channel statistics accounting for the $L-1$ other interfering BS, we will need to introduce a normalization factor to keep the interference bounded [13]. Since an increase of the number of users in an area with constant density implies a proportional increase of the path losses, we will assume that $\mathbb{E}\left[h_{i l}(t)^{2}\right]$ is in order of $\frac{1}{L}$. Alternatively, for notational convenience, we rename $h_{i l}(t)$ by $\frac{\sqrt{\beta} h_{i l}(t)}{\sqrt{L}}$ with $\mathbb{E}\left[h_{i l}(t)^{2}\right]=1$ and a normalization factor $\beta$ for network density. We then write here the interference as:

$$
I t_{l}(t)=\frac{\beta}{L-1} \sum_{k \neq j}\left|h_{k l}(t)\right|^{2} p_{k}(t)
$$

We assume that, as $L$ grows to infinity, the distribution function of the states variables of all BSs is turned into a continuum. Considering a continuum of players and a mean field game framework, we can reformulate the problem as a mean field game. The L-body initial problem can be simplified by focusing on a generic user and by considering a mean field interference. We now denote $Q(t)$, the packet size evolution of one generic user, which can be seen as a random variable with density the proportion of BSs in state $Q$ at time $t$. For the same reason, $h(t)$ will now define the channel for a generic user. We denote $m(t, X)$ the density of BSs in state $X=(Q, h)$ at time $t$. Similar to [4], we define the new interference term, with respect to the distribution $m_{t}$ :

$$
I t\left(t, m_{t}\right)=\beta \int_{Q} m(t, X)|h(t, X)|^{2} p(t, X) d Q d h
$$

where $p(t, Q, h)$ is the transmit power scheme used by a BS in state $X=(Q, h)$ at time $t$.

The minimization problem we now consider is based on the previous minimization problem (4) :

$$
\min _{p(t)} \mathbb{E}\left[\int_{0}^{T} p(t) d t+K(Q(T))\right]
$$

such that

$$
\begin{gathered}
d h(t)=\underbrace{(a(t) h(t)+b(t))}_{\alpha(t, h(t))} d t+\sigma_{b}(t) d W_{t} \\
d Q(t)=-w\left(t, p(t), h(t), m_{t}\right) d t
\end{gathered}
$$

where $w\left(t, p(t), h(t), m_{t}\right)=B \ln \left(1+\gamma\left(t, m_{t}, h(t), p(t)\right)\right)$ and $\gamma\left(t, m_{t}, h(t), p(t)\right)=\frac{p(t) h(t)}{\sigma_{n}^{2}+I t\left(t, m_{t}\right)}$. Finally, $Q(0), m_{0}$ and $h(0)$ are defined and initialize the game.

The running cost function to be considered in this section is defined by:

$$
v\left(t, X_{t}\right)=\mathbb{E}\left[\int_{t}^{T} p(u) d u+K(Q(T))\right]
$$

According to [8][9], the equivalent notion to the Nash equilibrium in the mean field game framework is the mean field equilibrium $\left[m^{*}\left(t, X_{t}\right), v^{*}\left(t, X_{t}\right)\right]$, solution to a coupled Hamilton-Jacobi-Bellman equation (from one single user point of view) and Fokker-Planck-Kolmogorov equation. First, the optimal trajectory $v^{*}(t, p(t))$ is solution to the HamiltonJacobi-Bellman (HJB) equation, adapted from (5):

$$
\begin{aligned}
& \quad 0=\min _{p(t)}\left[p(t)-w\left(t, p(t), h(t), m^{*}\left(t, X_{t}\right)\right) \partial_{Q} v\left(t, X_{t}\right)\right. \\
& \left.+\alpha(t) \partial_{h} v\left(t, X_{t}\right)\right]+\frac{1}{2} \sigma(t)^{2} \partial_{h h} v\left(t, X_{t}\right)-\partial_{t} v\left(t, X_{t}\right)
\end{aligned}
$$

with the terminal condition $v\left(T, X_{T}\right)=K(Q(T))$

This equation can be solved backwards and gives $v^{*}\left(t, X_{t}\right)$. The control strategy $p\left(t, X_{t}\right)$ is the argument of the min term of the previous equation, for a given trajectory of $v\left(t, X_{t}\right)$ :

$$
p\left(t, X_{t}\right)=\arg \min _{p(t)}\left[p(t)+\alpha(t) \partial_{h} v\left(t, X_{t}\right)\right.
$$




$$
\left.-w\left(t, p(t), h(t), m^{*}\left(t, X_{t}\right)\right) \partial_{Q} v\left(t, X_{t}\right)\right]
$$

The optimal trajectory for the distribution of users, $m^{*}\left(t, X_{t}\right)$, solves the Fokker-Planck-Kolmogorov equation, given by:

$$
\begin{gathered}
\partial_{h}\left[m\left(t, X_{t}\right) \alpha(t)\right]-\partial_{Q}\left[w\left(t, p^{*}\left(t, X_{t}\right), h(t), m\left(t, X_{t}\right)\right)\right] \\
-\frac{1}{2} \partial_{h h}\left[\sigma_{b}^{2} \cdot m\left(t, X_{t}\right)\right]+\partial_{t} m\left(t, X_{t}\right)=0
\end{gathered}
$$

Where $p^{*}(t, X)$ is as in (12) with $v(t, X)=v^{*}(t, X)$ and the initial distribution $m_{0}$ is given.

To summarize, a solution to the mean field game $\left[v^{*}\left(t, X_{t}\right), m^{*}\left(t, X_{t}\right)\right]$ can be obtained by solving the coupled equations (11) - (13). Once $v^{*}\left(t, X_{t}\right)$ is known, we have access to many system evolution information, which we study in the next section.

\section{Simulations: MANiPUlations AND RESUlts}

\section{A. Simplifying the model}

In this section, we assume that the channels are no longer time-varying, but are all constant and equal to 1 . The problem may appear greatly simplified, because the power strategies will not longer depend on the channel evolution but only on the initial packet size to be transmitted. Even though unrealistic in this setting, the scheduling problem can be solved numerically and shows some interesting hints on the system performance. In this case, the equivalent asymptotic fluid limit interference can be simplified:

$$
I t\left(t, m_{t}\right)=\beta \int_{Q} m(t, Q) p(t, Q) d Q
$$

where $m(t, Q)$ is now the density of users whose remaining packet size at time $t$ is $Q$ and $p(t, Q)$ is the transmit power adopted by such a user.

The minimization problem we now consider is :

$$
\min _{p(t)} \int_{0}^{T} p(t) d t+K(Q(T))
$$

such that

$$
d Q(t)=-w(t, p(t), m(t, Q)) d t
$$

where $w\left(t, p(t, Q), m_{t}\right)=B \ln (1+\gamma(t, m(t, Q), p(t, Q))$ and $\gamma\left(t, m_{t}, p(t)\right)=\frac{p(t)}{\sigma_{n}^{2}+I t\left(t, m_{t}\right)}$ for given $Q(0)$ and $m_{0}$.

Note that there is no longer any expectation, since the only random variable was due to channel uncertainty. The running cost function $v(t, Q)$ is now:

$$
v(t, Q)=\int_{t}^{T} p(u) d u+K(Q(T))
$$

A solution to the simplified mean field game $\left[m^{*}(t, Q), v^{*}(t, Q)\right]$ can be obtained by solving a simplified form of the previous coupled equations (11), (12), (13). The HJB (11) becomes:

$$
\partial_{t} v(t, Q)+\inf _{p(t)}\left[p(t)-B \ln \left(1+\gamma\left(t, m^{*}(t, Q)\right)\right) \partial_{Q} v(t, Q)\right]=0 .
$$

The control strategy $p(t, Q)$ is the argument of the min term of the previous equation, for a given trajectory of $v(t, Q)$ :

$p(t, Q)=\arg \inf _{p(t)}\left[p(t)-B \ln \left(1+\gamma\left(t, m^{*}(t, Q)\right)\right) \partial_{Q} v(t, Q)\right]$

i.e., $p(t, Q)=-\left(\sigma_{n}^{2}+I t\left(m_{t}^{*}\right)\right)+B \partial_{Q} v(t, Q)$.

Reusing the expression of $p(t, Q)$ in the HJB equation will provide its final version:

$$
\begin{gathered}
\left(B \ln \left(\frac{B \partial_{Q} v(t, Q)}{\sigma_{n}^{2}+I t\left(t, m_{t}\right)}\right)-1\right) \partial_{Q} v(t, Q) \\
+I t\left(t, m_{t}^{*}\right)-\partial_{t} v(t, Q)=0 .
\end{gathered}
$$

The previous FPK (13) can be simplified as:

$$
\partial_{t} m(t, Q)-\partial_{Q}[B \ln (1+\gamma(t, m(t, Q), p(t)) m(t, Q)]=0 .
$$

Reusing the expression of $p(t, Q)$ in the FPK equation provides its final version:

$$
\partial_{t} m_{t}=B \ln \left(\frac{B \partial_{Q} v^{*}(t, Q)}{\sigma_{n}^{2}+I t\left(t, m_{t}\right)}\right) \partial_{Q} m_{t}+m_{t} \frac{\partial_{Q Q} v^{*}(t, Q)}{\partial_{Q} v^{*}(t, Q)} .
$$

The problem now consists of a coupled equations in $m^{*}(t, Q)$ and $v^{*}(t, Q)$. Finding these two optimal solutions will allow us to recover both power strategies $p^{*}(t, Q)$ and packets size evolution $Q^{*}(t)$ for any user with initial size of packet $Q(0)$, given an initial distribution of packets among a large number of users $m_{0}$. The trajectory of such a user, will be obtained, thanks to equations (16) and (18).

\section{B. Results: observations and discussion}

We assume that $Q$ and $t$ both belong to a set $[0,1]$. We consider an initial distribution consisting of two truncated and scaled Gaussians of variance one: one is centered at 0.4 and the second one is centered at 0.75 . The final cost function $K$ (that strongly impacts the convergence of the fixed point algorithm) is chosen to be:

$$
K(Q(T))=\theta[\exp (\rho Q(T))-1] .
$$

The folloing results were obtained with $\rho=\theta=1$. We solve the system of coupled equations (HJB and FPK), by using a finite element method.

Fig. 1 represents $m^{*}(t, Q)$, i.e. the evolution of the distribution of packet sizes among the users across time. At $t=0$, we observe the two initial masses around 0.4 and 0.75 . In the end, almost every user has achieved its transmission as requested, only a little proportion of users have a remaining packet to receive, therefore accepting the penalty function. This suggests an appropriate choice of the penalty function.

Fig. 2 and 3 represent the evolution of $Q^{*}(t)$ and the cumulative power $\int_{0}^{T} p^{*}(t) d t$ for five users having five different initial packet sizes (equal to $0.2,0.4,0.6,0.8$ and 1 respectively). The power and the delay needed for a user to achieve its transmission are directly proportional to the initial packet size to be transmitted. We can observe that our system makes every user transmit at the beginning, whatever its initial packet size. Because of this, the users, whose initial packet 
were smaller, will achieve their transmissions first. This means that the number of active transmissions tends to get reduced when the deadline approaches. As observed by the increase of the slopes on Fig. 3, when active users disappear, the interference diminishes: the remaining transmitters will benefit from a better SINR and will rise their power of transmission.

\section{CONCLUSION}

In this article, we presented a dynamic $L$-body game framework to model the behaviour of BSs competing in the transmission of a data packet in time, at minimal cost. We turned our problem into a mean field game and proposed a consistent analysis of its mean field equilibrium. Through simulations, we highlighted some typical behaviors for our energy-efficient transmission scheme. We clearly observe a trade-off between distributing the power of the users on all the possible time slots, in order to reduce the global power consumption, and achieving some transmissions first, in order to reduce the number of users transmitting simultaneously, resulting in a better SINR and a better instantaneous data rate for the remaining users.

\section{REFERENCES}

[1] R.D.Yates "A framework for uplink power control in cellular radio systems", IEEE Journal on selected areas in communications, 1995

[2] J.Palicot, "Cognitive radio: an enabling technology for the green radio communications concept", proc. International Conference on Wireless Communications and Mobile Computing, 2009

[3] H.Tembine, S.Lasaulce, M.Jungers, "Joint power control-allocation for green cognitive wireless networks using mean field theory", IEEE proc. of the 5th Intl. Conf. CROWNCOM, 2010

[4] F.Mériaux and al., "Stochastic differential games and Green Power control", submitted to Dynamic Games and Applications, Feb. 2011

[5] R. Couillet, S.M.Perlaza, H.Tembine and M. Debbah, "A mean field game analysis of electric vehicles in the smart grid", IEEE International Conference on Computer Communications, Orlando, FL, USA, 2012.

[6] T.Basar and G.J.Olsder, "Dynamic Noncooperative game theory", vol. 160, Academic Press, pp 519, 1999

[7] B.K.Oksendal, "Stochastic Differential Equations: An Introduction with Applications", Universitext, 2003. Corr. 5th printing 2010

[8] J.M.Lasry and P.L.Lions "Jeux à champs moyens I - Le cas stationnaire", Comptes-rendus mathématiques, vol. 343, 2006

[9] J.M.Lasry and P.L.Lions "Jeux à champs moyens I - Horizon fini et contrle optimal", Comptes-rendus mathématiques, vol. 343, 2006

[10] K.Itô, "Multiple Wiener Integral", J.Math.Soc.Japan, vol.3, no.1, 1951

[11] L.Evans, "Partial differential equations", American Mathematical Society, 2010

[12] M.M.Olama, S.M.Djouadi and C.D.Charalambous, "Stochastic power control for time-varying long-term fading wireless networks", EURASIP

[13] A.Tulino, S.Verdú, "Random matrices and Wireless Communications. ", The essence of knowledge, 2004



Fig. 1. Optimal distribution of users $m^{*}(t, Q)$ whose packet size at time $t$ (x-axis) is $Q$ (y-axis).

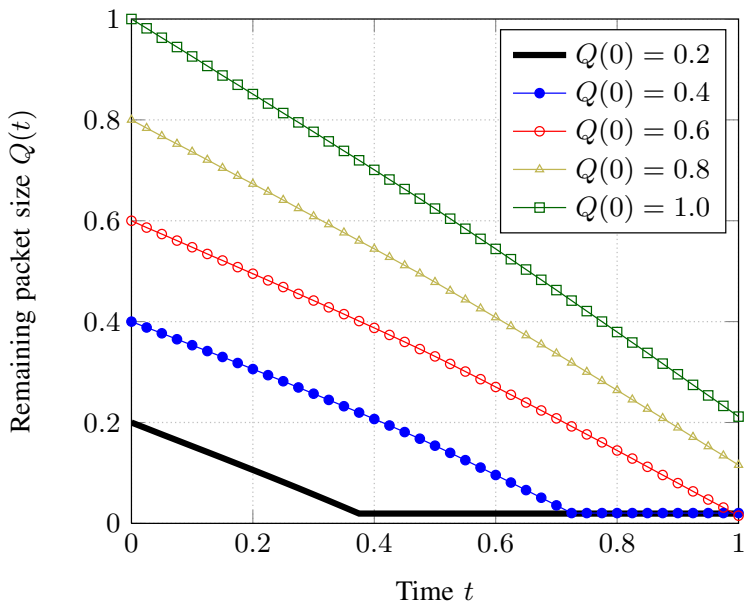

Fig. 2. Remaining packet size $Q(t)$ under optimal power $p^{*}\left(t, Q_{t}\right)$ for users with initial packet size $Q(0) \in\{0.2,0.4,0.6,0.8,1\}$.

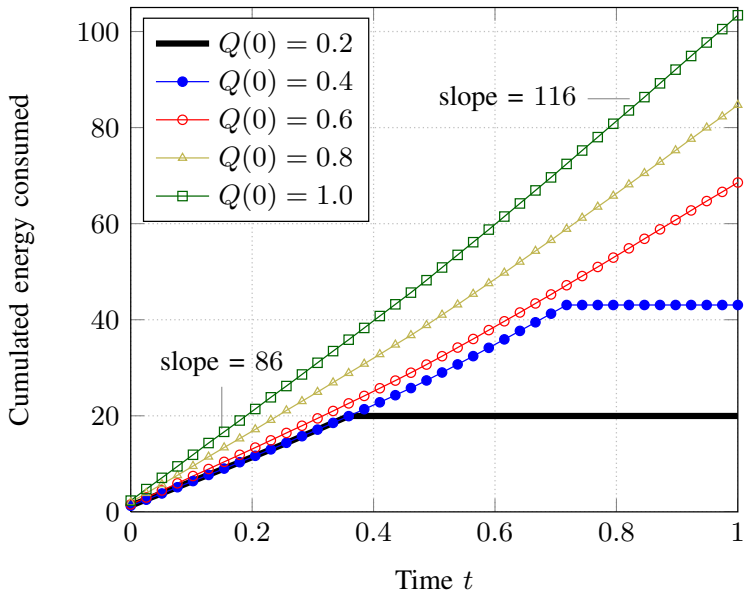

Fig. 3. Cumulated energy consumption $\int_{0}^{t} p^{*}(u, Q(u)) d u$ under optimal power policy $p^{*}(t, Q)$ for users with initial packet sizes $Q(0) \in$ $\{0.2,0.4,0.6,0.8,1\}$. 